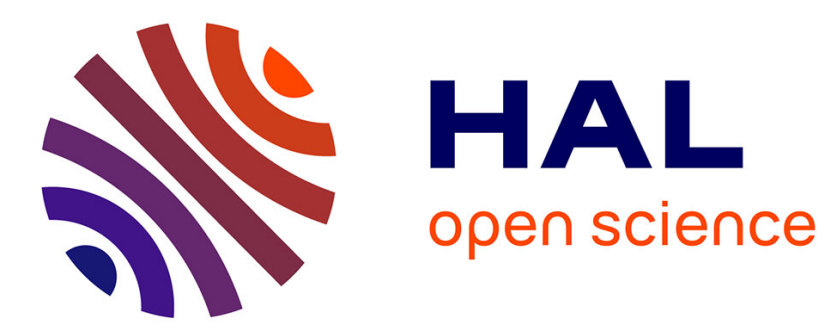

\title{
Oscillatory convective instabilities in nematic liquid crystals
}

\author{
H.N.W. Lekkerkerker
}

\section{To cite this version:}

H.N.W. Lekkerkerker. Oscillatory convective instabilities in nematic liquid crystals. Journal de Physique Lettres, 1977, 38 (14), pp.277-281. 10.1051/jphyslet:019770038014027700 . jpa-00231376

\section{HAL Id: jpa-00231376 https://hal.science/jpa-00231376}

Submitted on 1 Jan 1977

HAL is a multi-disciplinary open access archive for the deposit and dissemination of scientific research documents, whether they are published or not. The documents may come from teaching and research institutions in France or abroad, or from public or private research centers.
L'archive ouverte pluridisciplinaire HAL, est destinée au dépôt et à la diffusion de documents scientifiques de niveau recherche, publiés ou non, émanant des établissements d'enseignement et de recherche français ou étrangers, des laboratoires publics ou privés. 


\title{
OSCILLATORY CONVECTIVE INSTABILITIES IN NEMATIC LIQUID CRYSTALS
}

\author{
H. N. W. LEKKERKERKER \\ Faculteit van de Wetenschappen, Vrije Universiteit Brussel, 1050 Brussel, Belgium
}

(Reçu le 13 mai 1977, révisé le 13 juin 1977, accepté le 20 juin 1977)

\begin{abstract}
Résumé. - Nous présentons une extension, au cas de la convection oscillante, de la théorie de Dubois-Violette des instabilités hydrodynamiques dans les cristaux liquides nématiques soumis à gradient de température vertical.
\end{abstract}

\begin{abstract}
We present an extension of the Dubois-Violette theory of hydrodynamic instabilities in nematic liquid crystals subject to a vertical temperature gradient to the case of oscillatory convection.
\end{abstract}

Convective instabilities in nematic liquid crystals (L.C.'s) under vertical temperature gradients have been investigated both theoretically and experimentally [1-5]. The combination of the coupling between flow and orientation and the anisotropy of the heat diffusion constant gives rise to phenomena not found with vertical temperature gradients in isotropic fluids. The properties of stability with respect to stationary convection of L.C.'s under vertical temperature gradients are summarized below.

\section{TABLE I}

Stability properties with respect to stationary convection of nematics subject to vertical temperature gradients. $\chi_{\|}, \chi_{\perp}$ heat diffusion constants parallel and perpendicular to the director $\mathbf{n}$. Homeotropic orientation : $\mathbf{n}$ perpendicular to the limiting walls. Planar orientation : $\mathbf{n}$ parallel to the limiting walls.

$\begin{array}{lccccc} & \beta=-\frac{\partial T}{\partial z} & \chi_{\mathrm{a}}=\chi_{\|}-\chi_{\perp} & \begin{array}{c}\text { Homeotropic } \\ \text { orientation }\end{array} & \begin{array}{c}\text { Planar } \\ \text { orientation }\end{array} \\ & - & - & - & - & (\mathrm{P}) \\ \text { I. } & + & + & \text { Stable } & \text { Unstable } \\ \text { II. } & + & - & \text { Unstable } & \text { Stable } \\ \text { III. } & - & + & \text { Unstable } & \text { Stable } \\ \text { IV. } & - & - & \text { Stable } & \text { Unstable }\end{array}$

In the cases where the L.C. is unstable with respect to stationary convection, the temperature gradient at which the instability sets in is drastically reduced (by a factor of 100 to 1000 ) compared to the critical temperature gradient for an isotropic fluid under similar conditions. The explanation [1-4] for this feature is that the distortion of the alignment produced by the flow combined with the anisotropic heat diffu- sivity gives rise to a heat focusing effect which is either strongly stabilizing (IH, IVH, IIP, IIIP) or strongly destabilizing (IIH, IIIH, IP, IVP).

So far only the stability with respect to stationary convective motion has been considered. Here we study the stability with respect to oscillatory convection. The reason why one suspects that overstability may occur in this case is that the relaxation time of fluctuations in the orientation of the director $\left(K \xi q^{2}\right)^{-1}$ ( $K$ : elastic constant, $\xi$ : director relaxation constant, q : wave vector of the fluctuation) is long compared to the relaxation time of temperature fluctuations $\left(\chi q^{2}\right)^{-1}$. Under these conditions the stabilizing or destabilizing effect of the flow distortion induced heat focusing mechanism can be eliminated while retaining the destabilizing effect of a downward directed temperature gradient. The basic ideas of how this may happen are schematically illustrated in figure 1 for the case of a planar L.C. with $\chi_{\mathrm{a}}<0$. Consider an oscillatory velocity field with

$$
\begin{aligned}
\operatorname{div} \mathbf{v} & =0 \\
v_{y} & =0 \\
v_{z} & =v_{z}(\mathbf{q}) \cos \omega t \cos q_{x} x \sin q_{z} z .
\end{aligned}
$$

The frequency of oscillation is such that

$$
K \xi q^{2} \ll \omega \ll \chi q^{2} .
$$

Then it follows from the equations of nematodynamics [6] that the flow described by eq. (1) gives rișe to a distortion in the alignment having a $90^{\circ}$ phase lag with respect to the velocity field

$$
n_{z}=-\frac{C q}{\omega} v_{z}(q) \sin \omega t \sin q_{x} x \sin q_{z} z
$$




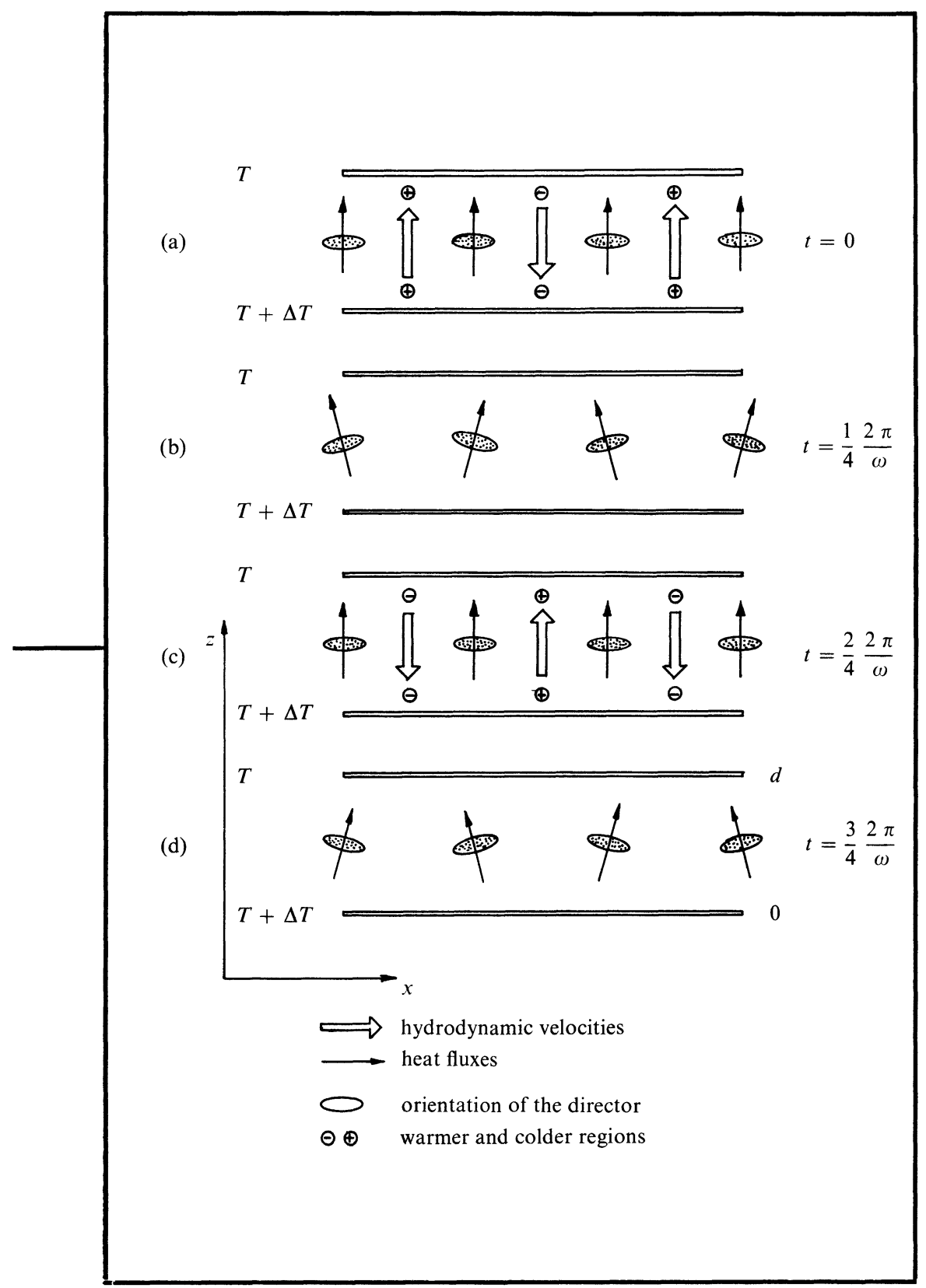

FIG. 1. - Oscillatory convective instability mechanism in a planar nematic liquid crystal with negative heat diffusion anisotropy.

Here $C$ is the coupling constant between flow and orientation. The result is that the heat focusing effect will be out of phase with respect to the velocity field and has little effect on the instability any more (see Fig. 1). On the other hand since $\omega \ll \chi q^{2}$ the temperature variations remain in phase with the velocity field. Thus qualitatively one expects that a L.C. heated from below will become unstable to oscillatory convective motion at roughly the same threshold as that for stationary convection in an isotropic fluid.

Oscillations will of course only be observed if the critical temperature gradient for the onset of oscillatory convective motion lies lower than the onset of stationary convection. One expects this to be the case for a homeotropic L.C. with $\chi_{\mathrm{a}}>0$ and a planar L.C. with $\chi_{\mathrm{a}}<0$.
We now determine the threshold for the onset of convection by a linear stability analysis in terms of normal modes. For the sake of variety we present the calculations for a homeotropic L.C. The variables that are coupled are

$$
\begin{aligned}
& \psi=(\operatorname{curl} \operatorname{curl} \mathbf{v})_{z} \\
& f=\frac{\partial n_{x}}{\partial x}
\end{aligned}
$$

and $\theta$, the fluctuation in the steady-state temperature distribution. We include the boundary conditions in an approximate manner such that the fluctuations can be written as a superposition of Fourier modes :

$[\psi(\mathbf{q}, t), f(\mathbf{q}, t), \theta(\mathbf{q}, t)] \cos q_{x} x \sin q_{z} z$ 
with

$$
q_{z}=n \cdot \frac{\pi}{d}, \quad n=1,2,3, \ldots
$$

Using the linearized nematodynamic equations $[6,7]$ one obtains the following equations of motion for the Fourier amplitudes

$$
\frac{\partial}{\partial t}\left[\begin{array}{l}
\psi(\mathbf{q}, t) \\
f(\mathbf{q}, t) \\
\theta(\mathbf{q}, t)
\end{array}\right]=-\mathbf{M}\left[\begin{array}{l}
\psi(\mathbf{q}, t) \\
f(\mathbf{q}, t) \\
\theta(\mathbf{q}, t)
\end{array}\right]
$$

where the hydrodynamic matrix $\mathbf{M}$ is given by

$\mathbf{M}=\left[\begin{array}{llc}\frac{v(\varphi) q^{2}}{\rho} & \frac{C(\varphi) K(\varphi) q^{4}}{\rho} & -g \alpha q_{x}^{2} \\ -C(\varphi) & K(\varphi) \xi q^{2} & 0 \\ -\frac{\beta}{q^{2}} & \chi_{\mathrm{a}} \beta & \chi(\varphi) q^{2}\end{array}\right]$

The various parameters appearing in the hydrodynamic matrix have the following meaning

$$
\begin{aligned}
v(\varphi) & =\frac{1}{2}\left(v_{1}+v_{2}\right) \sin ^{2} 2 \varphi+v_{3} \cos ^{2} 2 \varphi \\
\chi(\varphi) & =\chi_{\|} \cos ^{2} \varphi+\chi_{\perp} \sin ^{2} \varphi \\
C(\varphi) & =\frac{1}{2}(1+\lambda \cos 2 \varphi) \\
K(\varphi) & =K_{1} \sin ^{2} \varphi+K_{3} \cos ^{2} \varphi .
\end{aligned}
$$

Here $\varphi$ is the angle between $\mathbf{q}$ and $\mathbf{n}^{0}(/ / Z), v_{1}, v_{2}$ and $v_{3}$ are viscosity coefficients, $K_{1}$ and $K_{3}$ are elastic constants, $\lambda$ is the coupling constant between flow and orientation and $\alpha$ is the thermal expansivity.

The stability of the system is determined by the distribution of the roots of the characteristic polynomial $p(\mu)$ of $\mathbf{M}$

$$
p(\mu)=|\mu \mathbf{I}-\mathbf{M}|=\mu^{3}-a_{1} \mu^{2}+a_{2} \mu-a_{3} .
$$

The system will be stable if all roots have positive real parts. According to the theorem of RouthHurwith [8] this will be case if

$$
a_{1}>0, \quad a_{1} a_{2}-a_{3}>0, a_{3}>0 .
$$

On the other hand if

$$
a_{1}>0, a_{1} a_{2}-a_{3}>0, a_{3}=0,
$$

then $p(\mu)$ has two roots with positive real parts but one root that is equal to zero. Further if

$$
a_{1}>0, a_{1} a_{2}-a_{3}=0, a_{3}>0,
$$

then $p(\mu)$ has one positive real root and two purely imaginary roots $\left( \pm i \sqrt{a_{2}}\right)$. Thus the conditions given by eq. (8) determine the marginal state for the onset of stationary convection whereas the conditions given by eq. (9) determine the marginal state for the onset of oscillatory convection. Applying the condition $a_{3}=0$ one obtains (omitting angle dependence for simplicity)

$$
g \alpha \beta_{\mathrm{c}}^{\mathrm{s}} d^{4}=\frac{\left(\frac{v}{\rho}+\frac{C^{2}}{\rho \xi}\right) \chi}{(1-A)} \frac{q^{6} d^{4}}{q_{x}^{2}}
$$

where

$$
A=\frac{C \chi_{\mathrm{a}}}{K \xi} .
$$

The condition $a_{1} a_{2}-a_{3}=0$ yields

$$
\begin{aligned}
& g \alpha \beta_{\mathrm{c}}^{0} d^{4}= \\
& =\frac{\left(\frac{v}{\rho}+K \xi\right)\left(\frac{v}{\rho} K \xi+\frac{C^{2} K}{\rho}+\frac{v \chi}{\rho}+K \xi \chi+\chi^{2}\right)}{\left(\frac{v}{\rho}+\chi+C \chi_{\mathrm{a}}\right)} \frac{q^{6} d^{4}}{q_{x}^{2}} .
\end{aligned}
$$

The actual value of the threshold is obtained by setting

$$
q_{z}=q_{z, c}=\frac{\pi}{d}
$$

in the r.h.s. of eqs. (10) and (11) and minimizing the resulting expressions with respect to $\varphi$. This leads to rather complicated expressions but a tentative estimate of the threshold can be obtained by setting

$$
q_{x, c}=q_{z, c}=\frac{\pi}{d} .
$$

The calculations for the planar case do not present any additional difficulties and therefore we restrict ourselves to stating the results for this case. The system becomes unstable with respect to stationary convection when

$$
g \alpha \beta_{\mathrm{c}}^{\mathrm{s}} d^{4}=\frac{\left(\frac{v}{\rho}+\frac{C^{2}}{\rho \xi}\right)}{(1+A)} \frac{q^{6} d^{4}}{q_{x}^{2}}
$$

and unstable with respect to oscillatory convection when

$$
\begin{aligned}
& g \alpha \beta_{\mathrm{c}}^{0} d^{4}= \\
& =\frac{\left(\frac{v}{\rho}+K \xi\right)\left(\frac{v}{\rho} K \xi+\frac{C^{2} K}{\rho}+\frac{v \chi}{\rho}+K \xi \chi+\chi^{2}\right)}{\left(\frac{v}{\rho}+\chi-C \chi_{\mathrm{a}}\right)} \frac{q^{6} d^{4}}{q_{x}^{2}} .
\end{aligned}
$$

The (in)stability regions of L.C.'s that follow from eqs. (10) to (13) are schematically represented in figures $2 a$ and $2 b$. The thresholds for the onset of stationary convection obtained here are in agreement with previous work [1-4]. The thresholds for the onset of oscillatory convection have not been given before. 

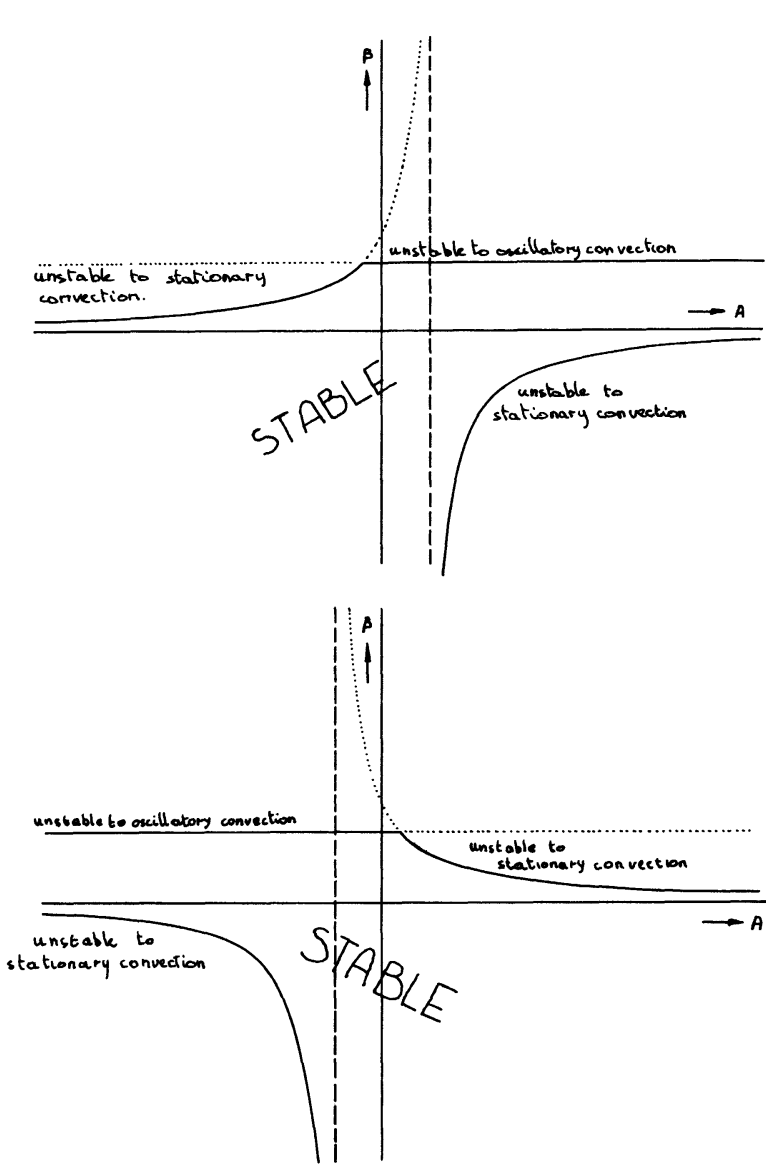

Fig. 2. - Thresholds and instability regions for nematics subject to vertical temperature gradients. a) Homeotropic orientation; b) Planar orientation.

Taking into account that

$$
\frac{v}{\rho} \gg \chi,\left(\frac{C^{2} K}{\rho}\right)^{1 / 2} \gg K \xi
$$

it follows that to a good approximation these thresholds (as given by eqs. (11) and (13)) can be written as

$$
g \alpha \beta_{\mathrm{c}}^{0} d^{4}=\frac{v}{\rho} \chi \frac{q^{6} d^{4}}{q_{x}^{2}} .
$$

Thus as predicted on the basis of qualitative considerations one finds that a L.C. heated from below becomes unstable to oscillatory convection at roughly the same threshold as that for stationary convection in an isotropic fluid [9].
So far no oscillatory convection has been observed in L.C.'s subject to vertical temperature gradients. This is not surprising since experiments so far [2-4] have been carried out with L.C. layers of maximum $1 \mathrm{~mm}$ thickness. Given that $\Delta T$ is limited to about $40^{\circ}$ (the range of liquid crystallinity) and given typical values of the thermodynamic derivatives and transport coefficients of nematics [6] one finds from eq. (14) that one has to work with layers $5 \mathrm{~mm}$ thick to trigger the oscillatory convective instability. The preparation of a well-oriented L.C. without defects of this thickness may indeed pose non-trivial problems.

The qualitative explanation illustrated in figure 1 for the possibility of oscillatory instabilities in nematics heated from below can, in essence, also be applied to other systems. For example in binary mixtures heated from below, the concentration gradient caused by the Soret effect has a stabilizing influence when the more dense component diffuses to the hot boundary. However, since the relaxation time of concentration fluctuations $\left(D q^{2}\right)^{-1}$ is long compared to the relaxation time of temperature fluctuations $\left(\chi q^{2}\right)^{-1}$, it is again possible to dephase the stabilizing effect of the concentration gradient by a velocity field with an oscillation frequency

$$
D q^{2} \ll \omega \ll \chi q^{2} .
$$

Oscillatory convection has indeed been observed in certain binary mixtures heated from below (for a recent review on the Rayleigh-Bénard instability in binary mixtures see ref. [10]).

To conclude, we have shown here using linear stability theory that the principle of exchange of stabilities does not always hold for L.C.'s subject to vertical temperature gradients, but that under certain conditions overstability may occur (see Fig. $2 a$ and $2 b$ ). It must be admitted that the linear theory given here does not contain a description of such interesting phenomena as subcritical finite amplitude instabilities and hysteresis (which has been observed in binary mixtures near the oscillatory marginal state [10]) for which linear theory is inadequate. Since even for isotropic fluids such problems have not been resolved completely we will not try to enter into a discussion of these points here.

Acknowledgments. - The author would like to thank I. Veretennicoff and E. Van Oost for fruitful discussions on the geometry of the zeros of polynomials and W. G. Laidlaw for useful discussions on instability mechanisms. The support of a NATO grant (No 806) is gratefully acknowledged. 


\section{References}

[1] Dubois-Violette, E., C. R. Hebd. Séan. Acad. Sci. B 273 (1971) 923.

Dubois-VioletTe, E., Solid State Commun. 14 (1974) 767.

[2] Guyon, E. and Pieranski, P., C. R. Hebd. Séan. Acad. Sci. B 274 (1972) 656.

[3] Pieranski, P., Dubois-Violette, E. and Guyon, E., Phys. Rev. Lett. 30 (1973) 736.

[4] Dubois-Violette, E., Guyon, E. and Pieranski, P., Mol. Cryst. Liq. Cryst. 26 (1974) 193.

[5] Barratt, P. J. and Sloan, D. M., J. Phys. A 9 (1976) 1987.
[6] De Gennes, P. G., The Physics of Liquid Crystals (Clarendon Press, Oxford) 1974, Ch. 5.

[7] Forster, D., Lubensky, T., Martin, P., Swift, J. and PerSHAN, P., Phys. Rev. Lett. 26 (1971) 1016.

[8] Gantmacher, F. R., Théorie des Matrices (Dunod, Paris) 1966, Tome 2, Ch. 15.

[9] ChandraseKhar, S., Hydrodynamic and Hydromagnetic Stability (Clarendon Press, Oxford) 1961, Ch. 2.

[10] Schechter, R. S., Velarde, M. G. and Platten, J. K., Adv. Chem. Phys. 26 (1974) 265. 